\title{
Protein tyrosine phosphatases in hypothalamic insulin and leptin signaling
}

Zhong-Yin Zhang ${ }^{1}$, Garron T. Dodd ${ }^{2}$ and Tony Tiganis ${ }^{2 *}$

${ }^{1}$ Department of Biochemistry and Molecular Biology, Indiana University School of Medicine, Indianapolis, IN, 46202-5126

${ }^{2}$ Department of Biochemistry and Molecular Biology, Monash University, Victoria 3800, Australia.

Running Title: Protein tyrosine phosphatases as drug targets in obesity

Keywords: Protein tyrosine phosphatases, obesity, type 2 diabetes, hypothalamus, leptin, insulin.

* Corresponding author: Tony Tiganis

Department of Biochemistry and Molecular Biology

Monash University, Victoria 3800, Australia

Tel 61399029332

Fax 61399029500

Email: Tony.Tiganis@monash.edu.

This is the author's manuscript of the article published in final edited form as:

Zhang, Z.-Y., Dodd, G. T., \& Tiganis, T. (2015). Protein Tyrosine Phosphatases in Hypothalamic Insulin and Leptin Signaling. Trends in Pharmacological Sciences, 36(10), 661-674. 


\section{ABSTRACT}

The hypothalamus is critical to the coordination of energy balance and glucose homeostasis. It responds to peripheral factors such as insulin and leptin that convey to the brain the degree of adiposity and the metabolic status of the organism. The development of leptin and insulin resistance in hypothalamic neurons appears to play a key role in the exacerbation of dietinduced obesity. In rodents, this has been attributed partly to the increased expression of the tyrosine phosphatases PTP1B and TCPTP that attenuate leptin and insulin signaling. Deficiencies in PTP1B and TCPTP in the brain, or specific neurons, promote insulin and leptin signaling and prevent diet-induced obesity, type 2 diabetes and fatty liver disease. Although targeting phosphatases and hypothalamic circuits remain challenging, recent advances indicate that such hurdles might be overcome. Here we focus on the roles of PTP1B and TCPTP in insulin and leptin signalling and explore their potential as therapeutic targets. 


\section{OBESITY EPIDEMIC}

Although obesity has been evident for millennia, its prevalence only began to rise in the last century. Between 1980 and 2013 the prevalence of overweight (BMI $>25 \mathrm{~kg} / \mathrm{m}^{2}$ ) and obese $\left(\mathrm{BMI}>30 \mathrm{~kg} / \mathrm{m}^{2}\right)$ individuals rose by $27.5 \%$ in adults and $47.1 \%$ in children, so that by 2013 there were 2.1 billion overweight and 671 million obese people worldwide. Excess body weight is a major and leading factor in overall disease burden and if left unabated could lead to decreases in life expectancy, particularly in developed nations [1]. Notably, the obesity epidemic is the single most important contributor to the development of type 2 diabetes (T2D), a major cause of obesity-associated morbidity and mortality [2, 3].

The environmental, genetic and socioeconomic factors underlying the development of obesity are complex. Its increasing prevalence highlights that dietary and lifestyle interventions alone are unlikely to be effective in combating the rising tide of obesity, and underscores the need for novel therapeutic approaches. To achieve this it is important that we understand the molecular perturbations that promote obesity and its complications. Large genome-wide association studies and meta-analyses suggest that $>20 \%$ of BMI variation may be accounted for by common genetic variation and have highlighted the principal importance of central nervous system (CNS) pathways in obesity [4]. CNS leptin and insulin pathways are important in the control of appetite, energy expenditure and glucose homeostasis [5] and molecules that antagonise leptin and insulin signaling in obesity may provide effective targets for therapeutic intervention. Prominent among such targets are the protein tyrosine phosphatases (PTPs) that dephosphorylate tyrosine-phosphorylated substrates to antagonise insulin and leptin-induced signaling [6, 7]. This review will focus on the role of classical PTPs, in particular PTP1B and TCPTP, in the CNS control of body weight and glucose homeostasis and explore approaches for inhibiting neuronal PTPs to combat obesity and T2D. 


\section{PTPs \& OBESITY}

\section{Leptin Signaling}

Leptin is a $16 \mathrm{kDa}$ cytokine that is produced by white adipocytes in approximate proportion to white adipose tissue (WAT) mass [8-10]. Leptin coordinates energy balance primarily via hypothalamic circuits that control feeding, movement, thermogenesis and glucose homeostasis [11-15]. Leptin's effects are additionally mediated by other regions of the brain, including for example the brainstem [16-18]. Leptin is transported across the blood brain barrier (BBB) by a saturable system [19]; transport into the cerebrospinal fluid (CSF) may be reliant on leptin signaling in median eminence tanycytes, specialised glial cells that extend from the third ventricle to the permeable fenestrated capillaries of the BBB [20] (Figure 2).

The arcuate nucleus (ARC), located in the basal part of the hypothalamus, is considered to have a fundamental role in sensing the global energy status of the organism, containing neurons that respond to peripheral stimuli such as leptin, insulin, ghrelin, glucose and free fatty acids [21-23]. The ARC contains two opposing neuronal populations: the appetite-suppressing proopiomelanocortin (POMC) [precursor of $\alpha$-melanocyte-stimulating hormone ( $\alpha$-MSH)] and the orexigenic neuropeptide Y (NPY) and AgRP-neuropeptide expressing neurons (Figure 2). POMC neuronal activation promotes $\alpha$-MSH release to agonise melanocortin- 4 receptors (MC4Rs) on 'second order' neurons in regions such as the paraventricular nucleus (PVN) of the hypothalamus, to control food intake, energy expenditure and metabolism [5]. Leptin depolarises (activates) POMC neurons to promote $\alpha$-MSH release, but hyperpolarises (inhibits) AgRP/NPY neurons and inhibits the release of AgRP and GABA that otherwise inhibit POMC neurons and antagonise $\alpha-\mathrm{MSH} / \mathrm{MC} 4 \mathrm{R}$ interactions in the PVN [5, 24-26] (Figure 2). The principal role of the melanocortin system in body weight control is underscored by the marked obesity evident in humans and rodents with null mutations in the genes encoding leptin, LEPRB, POMC or MCR4 [27]. 
Leptin signals via LEPR-B, a cell surface receptor that is associated with the protein tyrosine kinase (PTK) Janus-activated kinase 2 (JAK2) [28]. Leptin binding to LEPR-B activates JAK2, which in turn phosphorylates LEPR-B on tyrosines 985, 1077 and 1138. LEPRB Y1138 phosphorylation recruits STAT-3 via its SH2 domain [28]. STAT-3 is phosphorylated by JAK2 on Y705 to allow for STAT3 dimerisation and translocation to the nucleus to increase Pomc expression and inhibit Agrp expression [28, 29]. Leptin/LEPR-B also signals via JAK2 and the PI3K/AKT pathway to regulate neuronal excitability and AMPK activation and FoxO1 phosphorylation to alter neuropeptide expression and glucose metabolism [28-30]. These and other leptin signaling pathways elicit leptin's effects on energy balance and glucose homeostasis (Figure 3).

\section{Insulin Signaling}

Insulin is released from pancreatic $\beta$ cells following a rise in blood glucose and acts via the insulin receptor (IR) PTK and the PI3K/AKT pathway in liver, muscle and WAT to lower blood glucose levels and prevent post-prandial hyperglycemia. Insulin also acts in different regions of the brain to regulate, not only glucose homeostasis, but also energy balance, growth, reproduction, cognition and neural plasticity [31-36]. For example ICV insulin administration can repress food intake [32, 34, 37], inhibit WAT lypolysis [36, 38] and promote WAT browning and energy expenditure [39].

Insulin's CNS effects on whole-body glucose metabolism are mediated in part through the repression of hepatic glucose production [33, 40-42]. In the ARC, insulin binding results in IR activation and phosphorylation of insulin receptor substrate 2 (IRS-2) to recruit PI3K and activate the serine/threonine protein kinase AKT, which phosphorylates FoxO1 to increase POMC and decrease AgRP/NPY expression (Figures 2 and 3). Besides altering neuropeptide expression, insulin-induced PI3K signaling stimulates ATP sensitive $\mathrm{K}+$ channels $\left(\mathrm{K}_{\mathrm{ATP}}\right)$ to 
alter the electrical activity of POMC and AgRP neurons [43-45] (Figure 3). CNS insulin signaling lowers hepatic glucose production [33] and this can be blocked by the infusion of a $\mathrm{K}_{\mathrm{ATP}}$ blocker into the mediobasal hypothalamus, or by hepatic vagotomy [42]. Moreover genetic ablation of IR [31] or IRS-2 [46] in the brain, or the ICV inhibition of PI3K [33, 35], results in defective glucose homeostasis in mice. Insulin is thought to elicit its effects via ARC neurons, since IR deletion in AgRP neurons results in impaired repression of hepatic glucose production [41]. In lean humans, intranasal insulin delivery, so that insulin enters the CSF, increases hypothalamic and autonomic activity and enhances whole-body insulin sensitivity $[47,48]$. Thus the promotion of hypothalamic/ARC insulin sensitivity may provide a powerful means by which to independently combat the development of insulin resistance in obesity.

\section{PTPs and leptin signaling}

To date only a handful of protein tyrosine phosphatases (PTPs) have been implicated in leptin signaling, including SHP-2 that links LEPR-B to the Ras/MAPK pathway [49, 50], PTP1B [49, 51-54] and РTPE [55] that dephosphorylate JAK2, TCPTP [56] that dephosphorylates STAT3 [56], and phosphatase and tensin homologue (PTEN) [57] that attenuates PI3K signaling (Figure 3). Here we will focus on the roles of PTP1B and TCPTP since their elevated hypothalamic expression has been implicated in leptin resistance and dietinduced obesity $[51,56,58]$.

PTP1B dephosphorylates the Y1007/Y1008 site in the JAK2 PTK activation loop [59] (Figure 3). JAK2 Y1007/Y1008 phosphorylation is enhanced in $P t p n 1^{-/}$fibroblasts [52], and the PTP1B-D181A substrate-trapping mutant [60] can form a complex with Y1007/Y1008phosphorylated JAK2, but not tyrosine-phosphorylated LEPR-B or STAT3 in LEPR-Boverexpressing cells [53]. PTP1B's role in the control of energy homeostasis and hypothalamic leptin sensitivity was brought to light by the generation of $P t p n 1^{-/}$mice, which were resistant 
to diet-induced obesity [61, 62], and later by the deletion of PTP1B in neuronal and glial cells using the Nestin-Cre transgene [51]. Nestin-Cre;Ptpn1 $1^{f l f l}$ mice were resistant to diet-induced obesity, had reduced food intake and exhibited increased energy expenditure, ambulatory activity and leptin sensitivity [51]. The bulk of this phenotype was later recapitulated in mice lacking PTP1B in LEPR-B-expressing cells [54]. By contrast, mice lacking PTP1B in POMC neurons had a mild phenotype [39, 49]. Therefore, the overt effects of PTP1B deficiency on food intake and energy expenditure might be ascribed to other neurons, or other regions of the brain where PTP1B deficiency also promotes leptin sensitivity [54].

TCPTP also negatively regulates the leptin response [56] and is expressed in POMC [39] and AgRP neurons. TCPTP attenuates leptin signaling through the dephosphorylation of nuclear STAT3 [56] (Figure 3). TCPTP deletion in the brain (Nestin-Cre;Ptpn2 $2^{f l f l}$ ) promotes leptin-induced STAT3 signaling in the ARC and alters Pomc and Agrp expression and $\alpha$-MSH secretion [56]. Furthermore, Nestin-Cre;Ptpn $2^{f l / f l}$ mice exhibited an overall improvement in leptin sensitivity associated with decreased food intake, reduced adiposity and increased energy expenditure [56] that could be recapitulated by the ICV administration of a specific TCPTP inhibitor in C57BL/6 mice [56]. By contrast to PTP1B, TCPTP is redundant in leptin signaling in POMC neurons [39]; TCPTP deletion in POMC neurons (Pomc-Cre;Ptpn2 ${ }^{\text {fl/fl}}$ ) did not promote leptin-induced STAT3 phosphorylation or the effects on food intake or body weight [39]. Defining TCPTP's role in leptin signaling will require further investigation and an assessment of TCPTP function in other hypothalamic neurons and cell types.

\section{PTPs and insulin signaling}

Several PTPs have been shown to attenuate insulin signaling in peripheral tissues, but PTP1B is arguably the most critical [6]. PTP1B dephosphorylates the IR Y1162/Y1163 site and potentially IRS-1/2 to attenuate insulin signaling [6]. Ptpn1-/- mice, or liver- or muscle- 
specific PTP1B-deficient mice exhibit enhanced IR phosphorylation and signaling in liver and muscle and improved glucose homeostasis [51, 61-63].

There is evidence that PTP1B might also attenuate insulin signaling in the hypothalamus. PTP1B knockdown using antisense oligonucleotides enhances insulin signaling and promotes insulin-induced satiety to repress weight gain [64], whereas binge drinking has been shown to promote hypothalamic PTP1B expression to suppress the insulin-mediated repression of HGP [65]. However, PTP1B's role may be cell type-dependent, since PTP1B deletion in SF-1 neurons in the ventromedial hypothalamus enhances insulin signaling to suppress energy expenditure and promote weight gain in female mice [66], whereas PTP1B deletion in POMC neurons in the ARC has no effect on insulin signaling and glucose homeostasis, but enhances leptin signaling [39]. Rather, in POMC neurons, TCPTP may be the principal phosphatase attenuating insulin signaling [39]. TCPTP can dephosphorylate the IR Y1162/Y1163 site to attenuate insulin signaling [67] and TCPTP-deficiency in POMC neurons results in enhanced insulin-induced AKT Ser-473 phosphorylation and Pomc expression and improved glucose homeostasis [39]. Therefore, PTP1B and TCPTP may differentially contribute to ARC leptin and insulin signaling to regulate the melanocortin response (Figure 3).

\section{Leptin and Insulin Resistance}

Obesity and T2D are characterised by the development of insulin resistance and 'cellular' leptin resistance [68-70]. Insulin resistance involves the diminished responsiveness of insulin's key target tissues to insulin and has been attributed to varied factors including inflammation, ER stress and oxidative stress, which accompany the obese state [69-71]. Insulin resistance in liver, muscle and WAT is early hallmark of T2D and a driving factor in disease progression $[69,70]$. Hypothalamic insulin resistance also occurs in obesity [72-74], but the extent to which this contributes to the pathogenesis of T2D remains unclear. 
Whether 'cellular' leptin resistance initiates weight gain in obese humans remains incompletely understood. However, it is widely accepted that the increased adiposity associated with overfeeding promotes cellular leptin resistance in ARC neurons to exacerbate diet-induced obesity [68]. A number of mechanisms are thought to contribute to the development of cellular leptin resistance (and CNS insulin resistance). These include reduced transport across the BBB [75-77] and the enhancement of intracellular processes that attenuate LEPR-B signaling, including inflammation and ER stress [72, 78, 79] that drive the expression of negative regulatory molecules, such as suppressor of cytokine signaling 3 (SOCS3) and PTP1B [51-53, 58, 80, 81]. In rodents, genetic and pharmacological approaches that interfere with such negative regulatory processes promote LEPR-B signaling and thereby resistance to dietinduced obesity [51-53, 64, 79-83]. For example, overexpression of the unfolded protein response transcription factor $\mathrm{Xbp} 1 \mathrm{~s}$ in POMC neurons decreases $\mathrm{PTP} 1 \mathrm{~B}$ and SOCS3 expression, improves leptin and insulin sensitivity and liver metabolism and protects against diet-induced obesity [84]. Not surprisingly, deficiencies in neuronal PTP1B and SOCS3 promote leptin sensitivity and attenuate diet-induced obesity [51, 80-82].

The hyperleptinemia associated with the increased adiposity in obesity also promotes the feedback inhibition of leptin signalling by TCPTP [56]. Acute leptin administration increases hypothalamic TCPTP mRNA and protein expression and hypothalamic TCPTP levels are increased in obese C57BL/6 mice, but not leptin-deficient $\mathrm{Ob} / \mathrm{Ob}$ mice [56]. The increased expression of PTP1B and TCPTP in the hypothalamus is thought to result in the consolidated repression of the leptin (at the level of JAK2 and STAT3) and insulin signals, thereby exacerbating obesity and the development of T2D. Testament to this is that the combined deletion of PTP1B and TCPTP in neuronal and glial cells [56] or POMC neurons [39] results in the striking attenuation of diet-induced obesity (greater than that achieved by the deletion of either PTP alone), and markedly improved leptin sensitivity and glucose homeostasis. A direct 
assessment of PTP1B and TCPTP protein levels in the hypothalami of obese versus lean humans remains a challenge. However, it is known that in obese rodents, hypothalamic and skeletal muscle PTP1B protein levels are elevated as a consequence of inflammatory signalling [58]. Since obesity is associated with inflammation and PTP1B is elevated in human skeletal muscle in obesity [85], it is highly likely that hypothalamic PTP1B is also increased in obese humans. Furthermore, since leptin drives the expression of TCPTP in vitro and in vivo [56], it seems probably that hypothalamic TCPTP will also be elevated in obese hyperleptinemic individuals. Therefore, inhibiting both PTP1B and TCPTP in the ARC might be effective in ameliorating cellular leptin resistance and insulin resistance to combat diet-induced obesity.

\section{PTPs and the CNS control of WAT browning}

Recent studies have elucidated the principal role of the ARC in surveying peripheral energy reserves as conveyed by the combined actions of insulin and leptin and promoting energy expenditure through the sympathetic innervation of WAT (stores energy) and the increased formation of so-called 'beige’ fat (expends energy) [39], a process referred to as WAT browning [86] (Figure 4). Promoting WAT browning in rodents prevents diet-induced obesity and T2D [87, 88], whereas in humans, brown/beige fat depots are diminished in obesity [89]. In rodents, POMC neuronal activation increases WAT browning [39], whereas fasting and AgRP neuronal activation represses browning and energy expenditure [90], in keeping with the process being responsive to changes in energy status. PTP1B and TCPTP are expressed in both overlapping and anatomically distinct POMC neurons in the ARC and differentially contribute to the control of leptin and insulin signaling [39]. Their combined deficiency in POMC neurons and the promotion of both leptin and insulin signaling resulted in robust WAT browning, increased energy expenditure and afforded a remarkable resistance to diet-induced obesity and glucose intolerance and the development of hepatosteatosis [39]. Combined deficiencies in 
PTP1B and TCPTP in POMC neurons also increased interscapular brown adipose tissue (BAT) thermogenesis, but this was not due to the leptin/insulin synergy, but rather the promotion of leptin sensitivity [39]. Although POMC neurons in the nucleus of the solitary tracts of the hindbrain might also impact on BAT thermogenesis, TCPTP is not expressed in POMC neurons in this region of the brain [39], consistent with the synergistic effects on BAT thermogenesis being ascribed to the ARC. Irrespective, dampened responses of ARC POMC neurons and potentially AgRP neurons to circulating insulin and leptin, as a consequence of elevated levels of the inhibitory phosphatases PTP1B and TCPTP, might suppress WAT browning and energy expenditure and thereby exacerbate the development of obesity (Figure 4). Hence, the combinatorial inhibition of PTP1B and TCPTP in the hypothalamus/ARC may afford a means for not only promoting leptin-induced JAK2 and STAT3 signaling, but also the integrated response to leptin and insulin to increase WAT browning and energy expenditure to combat diet-induced obesity.

\section{PTP INHIBITORS}

PTP-based drug discovery has historically been shrouded with difficulties in attaining inhibitor selectivity and bioavailability, but in recent years these challenges have been convincingly met, through various approaches, including the generation of drugs targeting both the highly conserved phosphotyrosine (pTyr)-binding pocket [91] and adjacent substratebinding pockets, and through the generation of allosteric inhibitors [92], which can bind distant sites including disordered regions in PTP non-catalytic domains [93].

To address the issue of inhibitor potency and selectivity when targeting the pTyrbinding pocket [91], one can take advantage of amino acids and substrate-binding pockets that flank the active site and contribute to PTP substrate selectivity [94]. Accordingly, a novel paradigm was advanced for the design of potent and selective bivalent PTP inhibitors [95] by 
tethering appropriate diversity elements to a nonhydrolyzable pTyr mimetic to engage both the active site and nearby unique peripheral binding pockets. Bivalent PTP inhibitors are expected to possess enhanced affinity and selectivity due to additivity of free energy of binding and lack of structural similarity for second site interaction among different PTPs. To overcome the issue of bioavailability, several novel nonhydrolyzable pTyr mimetics have been discovered over the last few years that are sufficiently polar to bind the PTP active site, yet remain capable of efficiently crossing cell membranes [96, 97]. These pTyr mimetics interact in the desired inhibitory fashion with the PTP active site and the molecular scaffolds attached to them render the inhibitors PTP isozyme-selective. Recent studies using fragment-based and structureguided approaches to target both the PTP active site and adjacent less conserved pockets demonstrate that it is feasible to obtain PTP inhibitors with high affinity, selectivity, and excellent in vivo efficacy in animal models of oncology, diabetes/obesity, autoimmunity, and tuberculosis [98]. In addition to the active site-directed approach, another promising method that has been gaining significant traction in PTP drug discovery is targeting allosteric sites in PTPs that are less conserved, which has been demonstrated to be a good strategy for the development of inhibitors with high specificity and cell permeability [92].

In the following we highlight several inhibitors of PTP1B and TCPTP that have been used to further validate both phosphatases as therapeutic targets (Figure 5). Compound 1 was identified from a focused library approach with a $K_{\mathrm{i}}$ value of $2.4 \mathrm{nM}$ for PTP1B and several orders of magnitude selectivity in favor of PTP1B against a large panel of PTPs [99]. More importantly, compound $\mathbf{1}$ also displays $>10$-fold selectivity in favor of PTP1B over TCPTP, which is closely related to PTP1B (72\% catalytic domain sequence identify) [6]. Biochemical and structural studies show that compound $\mathbf{1}$ simultaneously occupies both the active site and a unique peripheral site in PTP1B [100]. As expected, compound 2, a cell permeable derivative of $\mathbf{1}$, is capable of augmenting insulin signaling and improving insulin sensitivity [101]. To 
more directly examine the role of PTP1B in leptin resistance, the effect of local inhibition of hypothalamic PTP1B was tested in 20-week old, leptin-resistant rats [102]. Acute ICV administration of compound 2 markedly improved the anorectic effects of leptin in 20-week old, leptin-resistant rats, indicating that hypothalamic PTP1B inhibition may be beneficial in alleviating leptin resistance. Compound 3 contains an isothiazolidinone (IZD) group as a pTyr mimetic and exhibits an IC 50 of $10 \mathrm{nM}$ for PTP1B and increased IR phosphorylation level in a dose-dependent manner [103]. Although compound 3 does not discriminate between PTP1B and TCPTP, it provides a proof of concept that heterocycles such as IZD can serve as highly efficacious pTyr mimetics. A dibenzo[b,d]furan mono-carboxylic acid derivative $\mathbf{4}$ was found to inhibit PTP1B with an $\mathrm{IC}_{50}$ value of $82 \mathrm{nM}$ and over 20-fold selectivity against TC-PTP [104]. Compound $\mathbf{4}$ was tested in vivo for anti-diabetic activity and showed significant efficacy in reducing body weight, fed- and fasting-state whole blood glucose, and plasma cholesterol levels in $\mathrm{Ob} / \mathrm{Ob}$ mice. Compound 5 represents another PTP1B inhibitor with a $K_{\mathrm{i}}$ of $0.2 \mu \mathrm{M}$ and greater than 46-fold selectivity over TCPTP and the receptor phosphatases CD45, and LAR [105]. Compound 5 is capable of increasing insulin-stimulated glucose uptake in L6 cells and insulin signaling in $o b / o b$ mice. Chronic administration compound 5 exerted a hypoglycemic effect without body weight gain.

In addition to the active site-directed PTP1B inhibitors described above, there is also substantial interest in an allosteric PTP1B inhibitor trodusquemine (also known as MSI-1436; compound 6), which was discovered serendipitously. Trodusquemine was originally discovered among a family of aminosterol compounds in the tissues of dogfish shark with broad-spectrum antimicrobial activity as well as potent appetite suppressant and antidiabetic properties [106108]. Subsequent investigation revealed that trodusquemine functioned as a potent noncompetitive inhibitor of PTP1B with a 200-fold preference (IC I0 $_{50} 1 \mu \mathrm{M}$ vs. $224 \mu \mathrm{M}$ ) over TCPTP [83]. The ability of trodusquemine to cause rapid and reversible weight loss in both 
genetic and diet-induced models of obesity has been further verified in additional studies [83, 109]. Through a series of elegant experiments, it was demonstrated that trodusquemine inhibits preferentially the full-length form of PTP1B, containing the regulatory C-terminal disordered fragment, thus revealing a novel mechanism of PTP1B allosteric inhibition [93]. It is anticipated that trodusquemine and/or its derivatives have the potential for treating T2D/obesity and other conditions with upregulated PTP1B activity, including breast cancer where PTP1B contributes to ErbB2/HER2-mediated tumorigenicity [93, 110].

Compared to PTP1B, much less drug discovery effort has been focused on TCPTP. Nevertheless, a fragment-based library synthesis and screening strategy led to the discovery of compound 7, an extremely potent and selective TCPTP inhibitor [111]. Compound 7 is a competitive inhibitor of TCPTP with a $K_{\mathrm{i}}$ of $4.3 \mathrm{nM}$ and is more than 200 -fold selective versus a panel of mammalian PTPs. Furthermore, an eight-fold selectivity for TCPTP over its closest homologue PTP1B is observed. Importantly, ICV administration of compound 7 enhances leptin-induced STAT3 Y705 phosphorylation, represses food intake and body weight and increases energy expenditure in mice [56] and promotes induced Pomc expression [39]. Collectively, the available data suggest that PTP1B and TCPTP inhibitors are capable of enhancing insulin and leptin sensitivity whether applied systemically, or locally to the brain.

\section{Concluding remarks}

Considerable progress has been made in defining the role of the phosphatases PTP1B and TCPTP in the CNS control of body weight. In particular recent studies have highlighted the potential for PTP1B and TCPTP to regulate the hypothalamic response to leptin and insulin and the promotion BAT thermogenesis, WAT browning and energy expenditure (). PTP1B and TCPTP levels are elevated in the hypothalamus in obesity, repressing leptin and insulin signaling and thereby perturbing energy balance (). Their inhibition may afford opportunities for therapeutic intervention and assist in combating the obesity epidemic. Although the hurdle 
of developing of selective PTP1B and TCPTP inhibitors has been demonstrably overcome, a significant albeit not insurmountable challenge remains in selectively targeting the brain. This is particularly important for TCPTP given its role in hematopoietic development and autoimmunity [112-115]. Although TCPTP heterozygous deficiency does not result in any overt immune phenotype [112], TCPTP heterozygoisty is sufficient to enhance T cell responses to self-antigen and autoimmunity, with age [115], in the context of lymphopenia [113] and in a mouse model of type 1 diabetes [114]. Despite this, recent work aimed at improving wholebody insulin sensitivity through the intranasal application of insulin and the enhancement of hypothalamic and parasympathetic outputs in humans $[47,48,74,116]$, suggests that selective CNS delivery of PTP1B/TCPTP inhibitors might be a viable option. Indeed the intranasal delivery of PTP1B/TCPTP inhibitors, directly into the CSF, may avoid the immune compartment and re-sensitise obese individuals to the beneficial CNS effects of insulin and leptin. Therefore dealing with the obesity and T2D epidemics with PTP inhibition might be just a puff away.

\section{BOX1: PTPs}

The PTP superfamily of enzymes comprises approximately 100 members that are defined by the active-site signature motif $\mathrm{HCX}_{5} R[6,117]$. The catalytic domains of classical PTPs comprise approximately 280 residues with 22 invariant and 42 highly conserved residues organised into 10 consensus motifs. These motifs contribute to conserved elements in the tertiary structure and can be involved in substrate recognition or catalysis. The cysteine and arginine residues within the $\mathrm{HCX}_{5} \boldsymbol{R}$ signature motif are essential for catalysis. The cysteine (PTP1B Cys-215) is located at the base of a deep cleft on the PTP surface. The depth of this cleft engenders specificity for phosphotyrosine- over phosphoserine/threonine-containing proteins, with the phosphatate moieties of the smaller phosphoserine and phosphothreonine 
unable to reach the cysteine at the base. The sulfur atom of the thiolate group of the cysteine residue serves as a nucleophile and attacks the substrate phosphate. An aspartate (PTP1B Asp181) on another conserved motif, the WPD loop, serves as a general acid to protonate the phenolic oxygen of the tyrosyl leaving group of the substrate to form a cysteine-phosphate intermediate. The conserved arginine (PTP1B Arg-221) in the signature motif contributes to substrate binding and stabilizes the cysteine-phosphate intermediate. In the second step of catalysis, the WPD loop aspartate acts as a general base and functions in concert with a highly conserved glutamine residue (PTP1B Gln-262) that coordinates a water molecule, to promote the hydrolysis of the cysteine-phosphate intermediate and release the phosphate.

The human genome encodes 37 (38 in rats and mice) classical, phosphotyrosine-specific phosphatases (Figure 1). The remaining approximately 65 members are defined as DSPs, based on their ability in vitro to dephosphorylate both tyrosine and serine/threonine phosphorylated substrates. The DSPs include the MKPs and PTEN, which dephosphorylates the 3' position in phosphatidylinositol (3,4,5)-triphosphate to attenuate PI3K signaling [6, 117$]$. The classical PTPs can exist as non-transmembrane cytosolic PTPs that have regulatory sequences flanking their catalytic domains, and transmembrane receptor-like PTPs that have transmembrane and extracellular domains with the potential to bind ligand $[6,117]$. The cytosolic PTPs include the prototypic PTP1B (PTPN1), which is targeted to the cytoplasmic face of the endoplasmic reticulum (ER), and TCPTP (PTPN2), which can exist as two variants targeted to the ER and nucleus by alternate non-catalytic C termini $[6,117]$. 


\section{GLOSSARY:}

AgRP; agouti-related peptide, a neuropeptide produced by AgRP/NPY neurons that promote feeding. AgRP inhibits POMC neurons and antagonises $\alpha$-MSH binding to MC4R.

AMPK; AMP-activated protein kinase

ARC; arcuate nucleus, an aggregation of neurons located in the mediobasal hypothalamus Cellular leptin resistance; refers to the diminished responsiveness of ARC neurons to circulating leptin

CNS; central nervous system

CSF; cerebrospinal fluid

DSPs; dual-specificity phosphatases

FoxO1; forkhead box O1

GABA; $\gamma$-aminobutyric acid

ICV; intracerebroventricular, within the ventricle of the brain

IRS; IR substrate

IR Y1162/Y1163 site; tyrosine phosphorylation site on the insulin receptor tyrosine kinase activation loop required for insulin receptor activation

JAK2; Janus-activated kinase 2

LEPR-B; long form of leptin receptor

MAPK; mitogen activated protein kinase

MC4R; melanocortin-4 receptor, receptor for $\alpha$-MSH

MKPs; MAPK phosphatases

$\alpha$-MSH; $\alpha$-melanocyte-stimulating hormone, a neurotransmitter derived from POMC that agonises MC4Rs on second order neurons in the PVN

NPY; neuropeptide Y, a neurotrasmitter

PTEN; phosphatase and tensin homologue

PI3K; phosphatidyinositol 3-kinase

POMC; proopiomelanocortin, a neuropeptide that is produced by POMC neurons and is the precursor of $\alpha$-MSH

PTPN1; gene encoding PTP1B

PTPN2; gene encoding TCPTP

PVN; paraventricular nucleus, a hypothalamic neuronal nucleus with afferent inputs from first order POMC and AgRP/NPY ARC neurons

SH2; protein domain that bind phosphotyrosine in a specific context

SOCS3; suppressor of cytokine signaling 3

STAT3; signal transducer and activator of transcription-3, a transcription factor that is phosphorylated by JAK-2 and mediates leptin-induced gene expression

substrate-trapping mutant; inactive PTP mutant that can bind substrates stably 


\section{ACKNOWLEDGEMENTS}

This work was supported by the National Health and Medical Research Council (NHMRC) of Australia to TT and the NIH to Z-YZ (RO1-CA69202). T.T. is a NHMRC of Australia Principal Research Fellow. 


\section{REFRENCES}

1 Kitahara, C.M., et al. (2014) Association between class III obesity (BMI of 40-59 kg/m2) and mortality: a pooled analysis of 20 prospective studies. PLoS Med 11, e1001673

2 Roglic, G. and Unwin, N. (2010) Mortality attributable to diabetes: estimates for the year 2010. Diabetes Res Clin Pract 87, 15-19

3 Johnson, A.M. and Olefsky, J.M. (2013) The origins and drivers of insulin resistance. Cell 152, 673-684

4 Locke, A.E., et al. (2015) Genetic studies of body mass index yield new insights for obesity biology. Nature 518, 197-206

5 Varela, L. and Horvath, T.L. (2012) Leptin and insulin pathways in POMC and AgRP neurons that modulate energy balance and glucose homeostasis. EMBO reports 13, 1079-1086

6 Tiganis, T. (2013) PTP1B and TCPTP - nonredundant phosphatases in insulin signaling and glucose homeostasis. FEBS $J$ 280, 445-458

7 St-Pierre, J. and Tremblay, M.L. (2012) Modulation of leptin resistance by protein tyrosine phosphatases. Cell Metab 15, 292-297

8 Considine, R.V., et al. (1996) Serum immunoreactive-leptin concentrations in normal-weight and obese humans. $N$ Engl J Med 334, 292-295

9 Frederich, R.C., et al. (1995) Leptin levels reflect body lipid content in mice: evidence for diet-induced resistance to leptin action. Nat Med 1, 1311-1314

10 Maffei, M., et al. (1995) Leptin levels in human and rodent: measurement of plasma leptin and ob RNA in obese and weight-reduced subjects. Nat Med 1, 1155-1161

11 Dhillon, H., et al. (2006) Leptin directly activates SF1 neurons in the VMH, and this action by leptin is required for normal body-weight homeostasis. Neuron 49, 191-203

12 Balthasar, N., et al. (2004) Leptin receptor signaling in POMC neurons is required for normal body weight homeostasis. Neuron 42, 983-991

13 Coppari, R., et al. (2005) The hypothalamic arcuate nucleus: a key site for mediating leptin's effects on glucose homeostasis and locomotor activity. Cell Metab 1, 63-72

14 Ring, L.E. and Zeltser, L.M. (2010) Disruption of hypothalamic leptin signaling in mice leads to early-onset obesity, but physiological adaptations in mature animals stabilize adiposity levels. J Clin Invest 120, 2931-2941

15 Dodd, G.T., et al. (2014) The thermogenic effect of leptin is dependent on a distinct population of prolactin-releasing peptide neurons in the dorsomedial hypothalamus. Cell Metab 20, 639-649

16 Scott, M.M., et al. (2011) Leptin receptor expression in hindbrain Glp-1 neurons regulates food intake and energy balance in mice. J Clin Invest 121, 2413-2421

17 Hayes, M.R., et al. (2010) Endogenous leptin signaling in the caudal nucleus tractus solitarius and area postrema is required for energy balance regulation. Cell Metab 11, 77-83

18 Myers, M.G., Jr., et al. (2009) The geometry of leptin action in the brain: more complicated than a simple ARC. Cell Metab 9, 117-123

19 Banks, W.A., et al. (1996) Leptin enters the brain by a saturable system independent of insulin. Peptides 17, 305-311

20 Balland, E., et al. (2014) Hypothalamic tanycytes are an ERK-gated conduit for leptin into the brain. Cell Metab 19, 293-301

21 Lam, T.K., et al. (2005) Hypothalamic sensing of circulating fatty acids is required for glucose homeostasis. Nat Med 11, 320-327

22 Parton, L.E., et al. (2007) Glucose sensing by POMC neurons regulates glucose homeostasis and is impaired in obesity. Nature 449, 228-232

23 Dietrich, M.O. and Horvath, T.L. (2013) Hypothalamic control of energy balance: insights into the role of synaptic plasticity. Trends Neurosci 36, 65-73 
24 Cowley, M.A., et al. (2001) Leptin activates anorexigenic POMC neurons through a neural network in the arcuate nucleus. Nature 411, 480-484

25 Tong, Q., et al. (2008) Synaptic release of GABA by AgRP neurons is required for normal regulation of energy balance. Nat Neurosci 11, 998-1000

26 van den Top, M., et al. (2004) Orexigen-sensitive NPY/AgRP pacemaker neurons in the hypothalamic arcuate nucleus. Nat Neurosci 7, 493-494

27 Coll, A.P., et al. (2004) Proopiomelanocortin and energy balance: insights from human and murine genetics. J Clin Endocrinol Metab 89, 2557-2562

28 Allison, M.B. and Myers, M.G., Jr. (2014) 20 years of leptin: connecting leptin signaling to biological function. $J$ Endocrinol 223, T25-35

29 Dagon, Y., et al. (2012) p70S6 Kinase Phosphorylates AMPK on Serine 491 to Mediate Leptin's Effect on Food Intake. Cell Metab 16, 104-112

30 Hill, J.W., et al. (2008) Acute effects of leptin require PI3K signaling in hypothalamic proopiomelanocortin neurons in mice. J Clin Invest 118, 1796-1805

31 Bruning, J.C., et al. (2000) Role of brain insulin receptor in control of body weight and reproduction. Science 289, 2122-2125

32 Obici, S., et al. (2002) Decreasing hypothalamic insulin receptors causes hyperphagia and insulin resistance in rats. Nat Neurosci 5, 566-572

33 Obici, S., et al. (2002) Hypothalamic insulin signaling is required for inhibition of glucose production. Nat Med 8, 1376-1382

34 Niswender, K.D., et al. (2003) Insulin activation of phosphatidylinositol 3-kinase in the hypothalamic arcuate nucleus: a key mediator of insulin-induced anorexia. Diabetes 52, 227231

35 Gelling, R.W., et al. (2006) Insulin action in the brain contributes to glucose lowering during insulin treatment of diabetes. Cell Metab 3, 67-73

36 Koch, L., et al. (2008) Central insulin action regulates peripheral glucose and fat metabolism in mice. J Clin Invest 118, 2132-2147

37 Woods, S.C., et al. (1979) Chronic intracerebroventricular infusion of insulin reduces food intake and body weight of baboons. Nature 282, 503-505

38 Scherer, T., et al. (2011) Brain insulin controls adipose tissue lipolysis and lipogenesis. Cell Metab 13, 183-194

39 Dodd, G.T., et al. (2015) Leptin and Insulin Act on POMC Neurons to Promote the Browning of White Fat. Cell 160, 88-104

40 Inoue, H., et al. (2006) Role of hepatic STAT3 in brain-insulin action on hepatic glucose production. Cell Metab 3, 267-275

41 Konner, A.C., et al. (2007) Insulin Action in AgRP-Expressing Neurons Is Required for Suppression of Hepatic Glucose Production. Cell Metab 5, 438-449

42 Pocai, A., et al. (2005) Hypothalamic K(ATP) channels control hepatic glucose production. Nature 434, 1026-1031

43 Spanswick, D., et al. (2000) Insulin activates ATP-sensitive K+ channels in hypothalamic neurons of lean, but not obese rats. Nat Neurosci 3, 757-758

44 Williams, K.W., et al. (2010) Segregation of acute leptin and insulin effects in distinct populations of arcuate proopiomelanocortin neurons. J Neurosci 30, 2472-2479

45 Qiu, J., et al. (2014) Insulin Excites Anorexigenic Proopiomelanocortin Neurons via Activation of Canonical Transient Receptor Potential Channels. Cell Metab 19, 682-693

46 Kubota, N., et al. (2004) Insulin receptor substrate 2 plays a crucial role in beta cells and the hypothalamus. J Clin Invest 114, 917-927

47 Heni, M., et al. (2014) Central insulin administration improves whole-body insulin sensitivity via hypothalamus and parasympathetic outputs in men. Diabetes 63, 4083-4088 
48 Dash, S., et al. (2015) Intranasal insulin suppresses endogenous glucose production in humans compared with placebo in the presence of similar venous insulin concentrations. Diabetes 64, 766-774

49 Banno, R., et al. (2010) PTP1B and SHP2 in POMC neurons reciprocally regulate energy balance in mice. $J$ Clin Invest 120, 720-734

50 Zhang, E.E., et al. (2004) Neuronal Shp2 tyrosine phosphatase controls energy balance and metabolism. Proc Natl Acad Sci U S A 101, 16064-16069

51 Bence, K.K., et al. (2006) Neuronal PTP1B regulates body weight, adiposity and leptin action. Nat Med 12, 917-924

52 Zabolotny, J.M., et al. (2002) PTP1B regulates leptin signal transduction in vivo. Dev Cell 2, 489-495.

53 Cheng, A., et al. (2002) Attenuation of leptin action and regulation of obesity by protein tyrosine phosphatase 1B. Dev Cell 2, 497-503.

54 Tsou, R.C., et al. (2014) Improved metabolic phenotype of hypothalamic PTP1B-deficiency is dependent upon the leptin receptor. Mol Metab 3, 301-312

55 Rousso-Noori, L., et al. (2011) Protein tyrosine phosphatase epsilon affects body weight by downregulating leptin signaling in a phosphorylation-dependent manner. Cell Metab 13, 562572

56 Loh, K., et al. (2011) Elevated hypothalamic TCPTP in obesity contributes to cellular leptin resistance. Cell Metab 14, 684-699

57 Plum, L., et al. (2006) Enhanced PIP3 signaling in POMC neurons causes KATP channel activation and leads to diet-sensitive obesity. J Clin Invest 116, 1886-1901

58 Zabolotny, J.M., et al. (2008) Protein-tyrosine phosphatase 1B expression is induced by inflammation in vivo. J Biol Chem 283, 14230-14241

59 Myers, M.P., et al. (2001) TYK2 and JAK2 are substrates of protein-tyrosine phosphatase 1B. J Biol Chem 276, 47771-47774.

60 Flint, A.J., et al. (1997) Development of "substrate-trapping" mutants to identify physiological substrates of protein tyrosine phosphatases. Proc. Natl. Acad. Sci. USA 94, 16801685

61 Elchebly, M., et al. (1999) Increased insulin sensitivity and obesity resistance in mice lacking the protein tyrosine phosphatase-1B gene. Science 283, 1544-1548

62 Klaman, L.D., et al. (2000) Increased energy expenditure, decreased adiposity, and tissuespecific insulin sensitivity in protein-tyrosine phosphatase 1B-deficient mice. Mol Cell Biol 20, 5479-5489

63 Delibegovic, M., et al. (2009) Liver-Specific Deletion of Protein-Tyrosine Phosphatase 1B (PTP1B) Improves Metabolic Syndrome and Attenuates Diet-Induced ER Stress. Diabetes 58, 590-599

64 Picardi, P.K., et al. (2008) Reduction of hypothalamic protein tyrosine phosphatase improves insulin and leptin resistance in diet-induced obese rats. Endocrinology 149, 38703880

65 Lindtner, C., et al. (2013) Binge drinking induces whole-body insulin resistance by impairing hypothalamic insulin action. Sci Transl Med 5, 170ra114

66 Chiappini, F., et al. (2014) Ventromedial hypothalamus-specific Ptpn1 deletion exacerbates diet-induced obesity in female mice. J Clin Invest 1243781-92, 3781-3792

67 Galic, S., et al. (2003) Regulation of insulin receptor signaling by the protein tyrosine phosphatase TCPTP. Mol Cell Biol 23, 2096-2108

68 Myers, M.G., Jr., et al. (2010) Obesity and leptin resistance: distinguishing cause from effect. Trends Endocrinol Metab 21, 643-651

69 Olefsky, J.M. and Glass, C.K. (2010) Macrophages, inflammation, and insulin resistance. Annu Rev Physiol 72, 219-246 
70 Samuel, V.T. and Shulman, G.I. (2012) Mechanisms for insulin resistance: common threads and missing links. Cell 148, 852-871

71 Ozcan, U., et al. (2004) Endoplasmic reticulum stress links obesity, insulin action, and type 2 diabetes. Science 306, 457-461

72 De Souza, C.T., et al. (2005) Consumption of a fat-rich diet activates a proinflammatory response and induces insulin resistance in the hypothalamus. Endocrinology 146, 4192-4199

73 Tschritter, O., et al. (2006) The cerebrocortical response to hyperinsulinemia is reduced in overweight humans: a magnetoencephalographic study. Proc Natl Acad Sci U S A 103, 1210312108

74 Gancheva, S., et al. (2015) Effects of intranasal insulin on hepatic fat accumulation and energy metabolism in humans. Diabetes

75 Caro, J.F., et al. (1996) Decreased cerebrospinal-fluid/serum leptin ratio in obesity: a possible mechanism for leptin resistance. Lancet 348, 159-161

76 Schwartz, M.W., et al. (1996) Cerebrospinal fluid leptin levels: relationship to plasma levels and to adiposity in humans. Nat Med 2, 589-593

77 Van Heek, M., et al. (1997) Diet-induced obese mice develop peripheral, but not central, resistance to leptin. J Clin Invest 99, 385-390

78 Zhang, X., et al. (2008) Hypothalamic IKKbeta/NF-kappaB and ER stress link overnutrition to energy imbalance and obesity. Cell 135, 61-73

79 Ozcan, L., et al. (2009) Endoplasmic reticulum stress plays a central role in development of leptin resistance. Cell Metab 9, 35-51

80 Briancon, N., et al. (2010) Combined neural inactivation of SOCS-3 and PTP-1B reveal additive, synergistic, and factor-specific roles in the regulation of body energy balance. Diabetes 59, 3074-3084

81 Kievit, P., et al. (2006) Enhanced leptin sensitivity and improved glucose homeostasis in mice lacking suppressor of cytokine signaling-3 in POMC-expressing cells. Cell Metab 4, 123132

82 Howard, J.K., et al. (2004) Enhanced leptin sensitivity and attenuation of diet-induced obesity in mice with haploinsufficiency of Socs3. Nat Med 10, 734-738

83 Lantz, K.A., et al. (2010) Inhibition of PTP1B by trodusquemine (MSI-1436) causes fatspecific weight loss in diet-induced obese mice. Obesity 18, 1516-1523

84 Williams, K.W., et al. (2014) Xbp1s in Pomc Neurons Connects ER Stress with Energy Balance and Glucose Homeostasis. Cell Metab 20, 471-82.

85 Ahmad, F., et al. (1997) Alterations in skeletal muscle protein-tyrosine phosphatase activity and expression in insulin-resistant human obesity and diabetes. J Clin Invest 100, 449-458

86 Rosen, E.D. and Spiegelman, B.M. (2014) What we talk about when we talk about fat. Cell 156, 20-44

87 Seale, P., et al. (2011) Prdm16 determines the thermogenic program of subcutaneous white adipose tissue in mice. $J$ Clin Invest 121, 96-105

88 Cohen, P., et al. (2014) Ablation of PRDM16 and Beige Adipose Causes Metabolic Dysfunction and a Subcutaneous to Visceral Fat Switch. Cell 156, 304-316

89 Ouellet, V., et al. (2011) Outdoor temperature, age, sex, body mass index, and diabetic status determine the prevalence, mass, and glucose-uptake activity of 18F-FDG-detected BAT in humans. J Clin Endocrinol Metab 96, 192-199

90 Ruan, H.B., et al. (2014) O-GlcNAc Transferase Enables AgRP Neurons to Suppress Browning of White Fat. Cell 159, 306-317

91 Barr, A.J., et al. (2009) Large-scale structural analysis of the classical human protein tyrosine phosphatome. Cell 136, 352-363

92 Wiesmann, C., et al. (2004) Allosteric inhibition of protein tyrosine phosphatase 1B. Nat Struct Mol Biol 11, 730-737 
93 Krishnan, N., et al. (2014) Targeting the disordered C terminus of PTP1B with an allosteric inhibitor. Nat Chem Biol 10, 558-566

94 Zhang, Z.Y. (2002) Protein tyrosine phosphatases: structure and function, substrate specificity, and inhibitor development. Annu Rev Pharmacol Toxicol 42, 209-234

95 Puius, Y.A., et al. (1997) Identification of a second aryl phosphate-binding site in proteintyrosine phosphatase 1B: a paradigm for inhibitor design. Proc Natl Acad Sci U S A 94, 1342013425

96 Combs, A.P. (2010) Recent advances in the discovery of competitive protein tyrosine phosphatase 1B inhibitors for the treatment of diabetes, obesity, and cancer. J Med Chem 53, 2333-2344

$97 \mathrm{He}, \mathrm{Y}$., et al. (2012) Bicyclic benzofuran and indole-based salicylic acids as protein tyrosine phosphatase inhibitors. Bioorg Med Chem 20, 1940-1946

$98 \mathrm{He}, \mathrm{R}$. , et al. (2013) Small molecule tools for functional interrogation of protein tyrosine phosphatases. FEBS $J$ 280, 731-750

99 Shen, K., et al. (2001) Acquisition of a specific and potent PTP1B inhibitor from a novel combinatorial library and screening procedure. J Biol Chem 276, 47311-47319

100 Sun, J.P., et al. (2003) Crystal structure of PTP1B complexed with a potent and selective bidentate inhibitor. J Biol Chem 278, 12406-12414

101 Xie, L., et al. (2003) Cellular effects of small molecule PTP1B inhibitors on insulin signaling. Biochemistry 42, 12792-12804

102 Morrison, C.D., et al. (2007) Increased hypothalamic protein tyrosine phosphatase 1B contributes to leptin resistance with age. Endocrinology 148, 433-440

103 Combs, A.P., et al. (2006) Potent benzimidazole sulfonamide protein tyrosine phosphatase $1 \mathrm{~B}$ inhibitors containing the heterocyclic (S)-isothiazolidinone phosphotyrosine mimetic. $J$ Med Chem 49, 3774-3789

104 Lakshminarayana, N., et al. (2010) Synthesis and evaluation of some novel dibenzo[b,d]furan carboxylic acids as potential anti-diabetic agents. Eur J Med Chem 45, 37093718

105 Fukuda, S., et al. (2010) Pharmacological profiles of a novel protein tyrosine phosphatase 1B inhibitor, JTT-551. Diabetes Obes Metab 12, 299-306

106 Moore, K.S., et al. (1993) Squalamine: an aminosterol antibiotic from the shark. Proc Natl Acad Sci U S A 90, 1354-1358

107 Rao, M.N., et al. (2000) Aminosterols from the dogfish shark Squalus acanthias. J Nat Prod 63, 631-635

108 Zasloff, M., et al. (2001) A spermine-coupled cholesterol metabolite from the shark with potent appetite suppressant and antidiabetic properties. Int J Obes Relat Metab Disord 25, 689697

109 Ahima, R.S., et al. (2002) Appetite suppression and weight reduction by a centrally active aminosterol. Diabetes 51, 2099-2104

110 Julien, S.G., et al. (2007) Protein tyrosine phosphatase 1B deficiency or inhibition delays ErbB2-induced mammary tumorigenesis and protects from lung metastasis. Nat Genet 39, 338346

111 Zhang, S., et al. (2009) Acquisition of a potent and selective TC-PTP inhibitor via a stepwise fluorophore-tagged combinatorial synthesis and screening strategy. J Am Chem Soc 131, 13072-13079

112 You-Ten, K.E., et al. (1997) Impaired bone marrow microenvironment and immune function in T cell protein tyrosine phosphatase-deficient mice. $J$ Exp Med 186, 683-693

113 Wiede, F., et al. (2014) PTPN2 attenuates T-cell lymphopenia-induced proliferation. Nat Commun 5, 3073 
114 Wiede, F., et al. (2014) PTPN2 restrains CD8(+) T cell responses after antigen crosspresentation for the maintenance of peripheral tolerance in mice. $J$ Autoimmun 53, 105-114

115 Wiede, F., et al. (2011) T cell protein tyrosine phosphatase attenuates T cell signaling to maintain tolerance in mice. $J$ Clin Invest 121, 4758-4774

116 Born, J., et al. (2002) Sniffing neuropeptides: a transnasal approach to the human brain. Nat Neurosci 5, 514-516

117 Tonks, N.K. (2013) Protein tyrosine phosphatases--from housekeeping enzymes to master regulators of signal transduction. FEBS J 280, 346-378 


\section{FIGURE LEGENDS}

Figure 1. Schematic of classical PTPs. The classical PTPs catalyse the dephosphorylation of tyrosyl phosphorylated protein substrates and include 38 family members (37 in humans) that are subdivided into non-transmembrane, cytosolic PTPs (17 members) and transmembrane receptor-like PTPs (21 members). The non-transmembrane, cytosolic PTPs have regulatory, substrate interaction and targeting sequences flanking their single catalytic domains, whereas the transmembrane receptor-like PTPs have one or two catalytic domains (the membrane distal PTP domain is often inactive or has residual activity and referred to as a PTP pseudophosphatase domain), in some cases juxtamembrane domains, single-pass transmembrane domains and variable extracellular domains, some of which bind ligand. The catalytic domains of all classical PTPs are characterised by the [I/V]HCXXGXXR[S/T] signature motif, in which the Cys and Arg residues are essential for catalytic activity. Abbreviations: BRO-1, BRO-1 homology; CAH, carbonic anhydrase-like; Cad, cadherin-like juxtamembrane sequence; FERM, FERM (4.1/ezrin/radixin/moesin) domain; FN, fibronectin type III-like domain; Gly, glycosylated; HD, histidine domain; Ig, immunoglobulin domain; KIM, kinase-interacting motif; MAM, mephrin/A5/ $\mu$ domain; Pro, proline-rich; RGDS, RGDSadhesion recognition motif; SEC14, SEC14/cellular retinaldehyde-binding protein-like; SH2, Src homology 2.

Figure 2. CNS insulin and leptin signaling in energy expenditure and glucose homeostasis. The circulating adipokine leptin and the hormone insulin communicate to the ARC and hypothalamus the organisms peripheral energy reserves and metabolic status. Leptin and insulin are transported to the hypothalamus/ARC across a blood brain barrier and specialised glial cells known as tanycytes. Within the ARC leptin and insulin engage their respective receptors (LEPR-B, IR) on two opposing neuronal populations known as POMC and 
AgRP/NPY neurons. POMC neuronal activation represses food intake and promotes expenditure whereas AgRP stimulates food intake. The neuropeptide POMC is processed to $\alpha-$ MSH and acts on the MCR4 receptor on second order hypothalamic neurons in the PVN. AgRP/NPY inhibit POMC neuronal activation and antagonise the $\alpha$-MSH/MCR4 interaction. These signals are propagated to the rest of the brain and peripheral tissue such as WAT, BAT, pancreas, muscle and the liver to regulate food intake, whole-body energy expenditure and metabolism. Abbreviations: $\alpha-\mathrm{MSH}$, alpha-melanocyte-stimulating hormone; AgRP, Agoutirelated peptide; ARC, Arcuate nucleus; MC4R, Melanocortin 4 receptor; POMC, proopiomelanocortin; PVN; Paraventricular nucleus; NPY, neuropeptide Y.

Figure 3. PTPs attenuate CNS insulin and leptin signalling. Leptin and insulin signal in hypothalamic neurons via the JAK2/STAT3 and JAK2/IRS-2/PI3K pathways to promote the expression of the anorexigenic (appetite suppressant) neuropeptide POMC and repress the expression of the orexigenic neuropeptides AgRP and NPY. Leptin and insulin also signal via the JAK2/IRS-2/PI3K pathway 1 ) to stimulate ATP sensitive $\mathrm{K}+$ channels ( $\left.\mathrm{K}_{\mathrm{ATP}}\right)$ to alter the electrical activity of POMC and AgRP neurons, and 2) to activate the protein kinase AKT to regulate glucose metabolism. These pathways are attenuated by the classical PTPs PTP1B, PTPe and TCPTP, the DSP PTEN, which dephosphorylates the lipid products of PI3K, and by SOCS3. PTP1B and TCPTP act together to attenuate leptin-induced JAK2/STAT3 signalling with PTP1B dephosphorylating the Y1007/Y1008 PTK activation loop site on JAK2 which is associated with the leptin receptor, and TCPTP the Y705 phosphorylation site on STAT3 that is required for STAT3 dimerisation and translocation to the nucleus where it mediates effects on gene expression. PTP1B and TCPTP also dephosphorylate the Y1162/Y1163 PTK activation loop site on the insulin receptor to attenuate insulin signaling. 
Figure 4. CNS control of WAT browning and energy expenditure. In periods of positive energy balance when leptin and insulin levels are elevated, leptin and insulin act synergistically on POMC neurons to promote the conversion of white fat into beige fat, referred to as WAT browning, and the expenditure of energy to maintain body weight. In obesity when hypothalamic PTP1B and TCPTP levels are elevated, leptin and insulin signalling are attenuated resulting in diminished WAT browning, decreased energy expenditure and increased weight gain.

Figure 5. PTP1B and TCPTP inhibitors. The figure depicts the structures of PTP1B inhibitors, compounds 1 [100], 2 [101], 4 [104], 5 [105], and $\mathbf{6}$ (also known as trodusquemine and MSI1436) [93], the TCPTP inhibitor compound 7 [111] and a PTP1B and TCPTP dual inhibitor compound 3 [103]. 
Figure 1

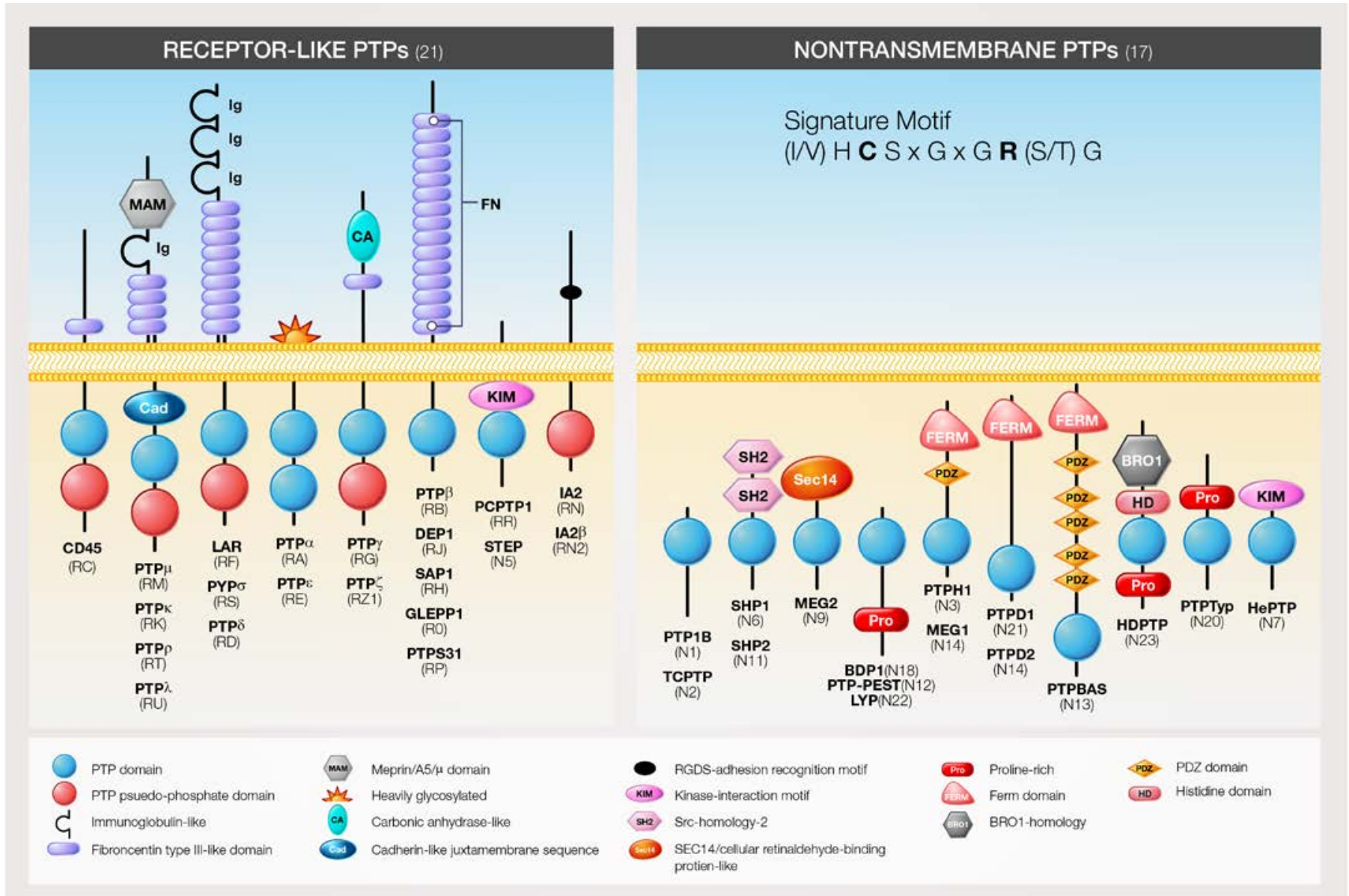


Figure 2

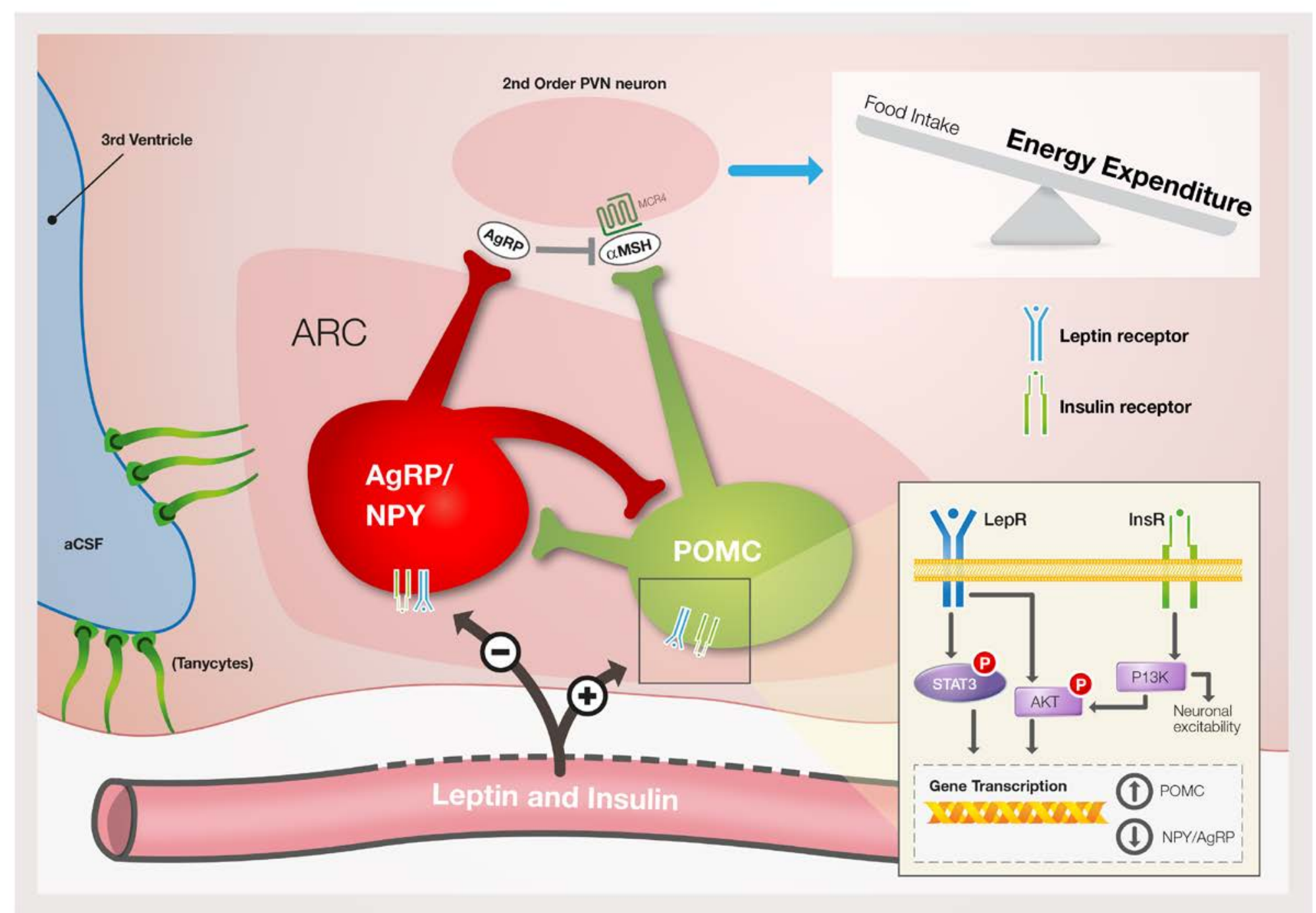


Figure 3.

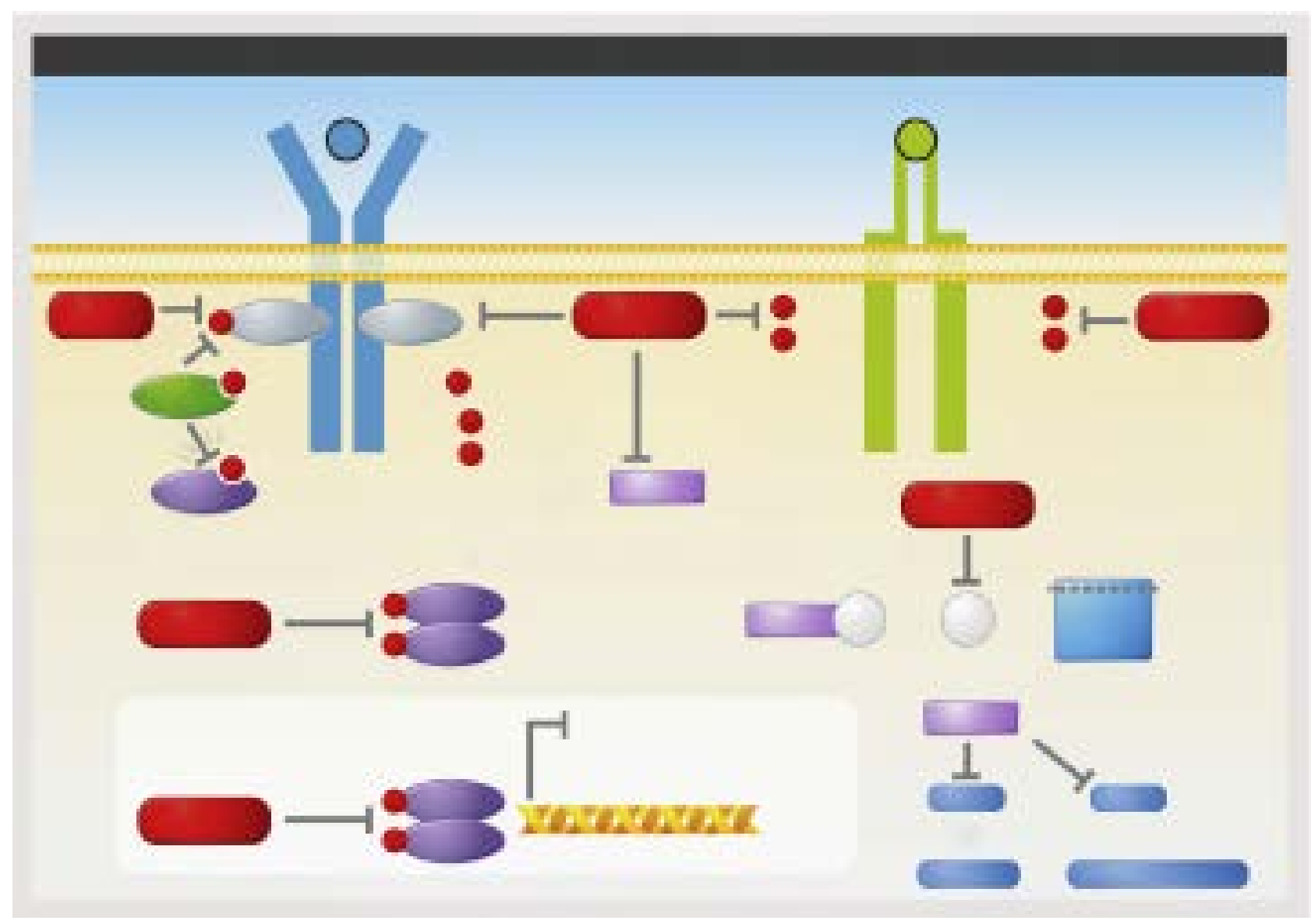


Figure 4.

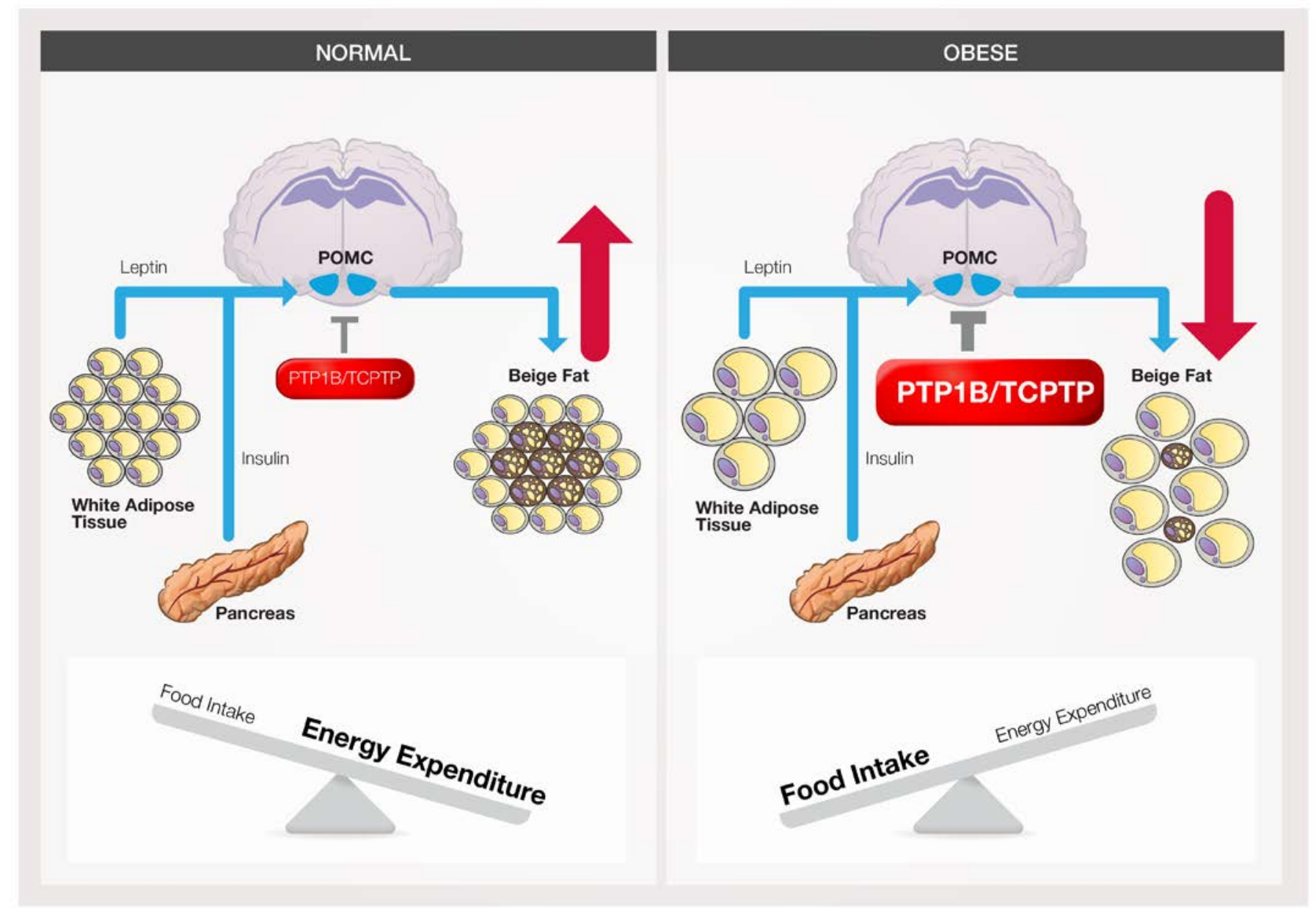


Figure 5.<smiles>CCCCCCCCCCCCCCC(=O)N[C@@H](Cc1ccc(C(O)(F)F)cc1)C(=O)N[C@H](CC(=O)O)C(=O)N[C@@H](Cc1ccc(C(F)(F)[PH](O)(O)O)cc1)C(N)=O</smiles>

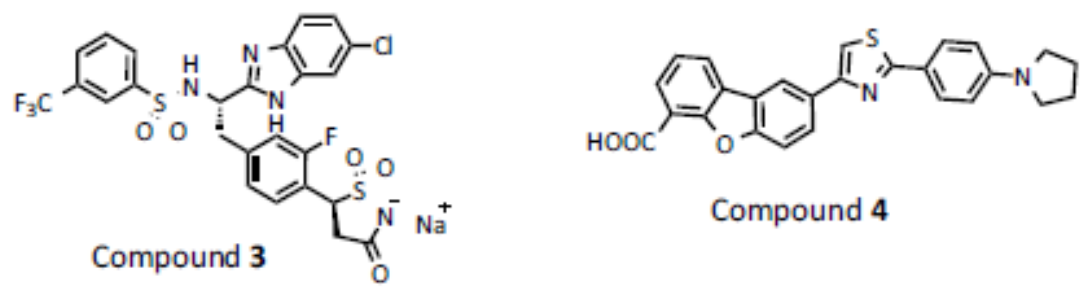<smiles></smiles>

Compound 5<smiles>CC(C)C(CCC(C)C1CCC2C(O)CC3CC(NCCCNCCCCNCCCN)CCC3(C)C2CC1C)C(=O)O</smiles>

Compound 6, Trodusquemine

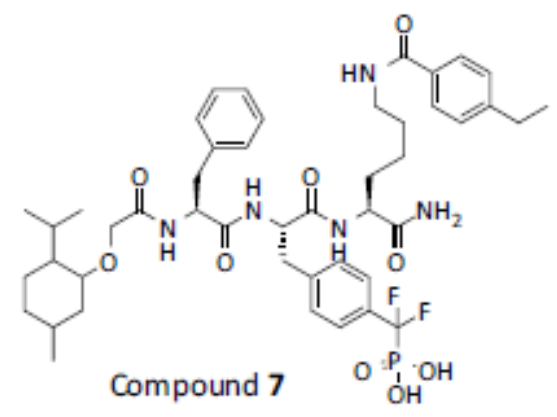

